\title{
The management of anterior mediastinal nodules that are incidentally found on CT
}

\author{
Yoshiyuki Ozawa \\ Department of Radiology, Nagoya City University, Graduate School of Medical Sciences, Mizuho-ku, Nagoya, Japan \\ Correspondence to: Yoshiyuki Ozawa, MD, PhD. Department of Radiology, Nagoya City University, Graduate School of Medical Sciences, 1 \\ Kawasumi, Mizuho-cho, Mizuho-ku, Nagoya 467-8601, Japan. Email: ykiooster@gmail.com. \\ Provenance: This is an invited Editorial commissioned by Editor-in-Chief Changqing Pan (Shanghai Chest Hospital Affiliated to Shanghai Jiao Tong \\ University, Shanghai, China). \\ Comment on: Yoon SH, Choi SH, Kang CH, et al. Incidental Anterior Mediastinal Nodular Lesions on Chest CT in Asymptomatic Subjects. J Thorac \\ Oncol 2018;13:359-66.
}

Received: 18 September 2018; Accepted: 29 November 2018; Published: 07 December 2018.

doi: $10.21037 /$ shc. 2018.11 .09

View this article at: http://dx.doi.org/10.21037/shc.2018.11.09

We sometimes encounter problems during the management of patients with anterior mediastinal nodular lesions that were incidentally detected on screening chest computed tomography (CT). As the utilization of CT imaging in clinical practice has increased, asymptomatic anterior mediastinal nodular lesions are being detected more frequently. Thymic epithelial tumors (TET) are representative of such lesions; however, there are a few reports about the prevalence and incidence of anterior mediastinal masses that are incidentally detected on chest CT (1-3). An article published by Yoon et al. in the Fournal of Thoracic Oncology described the prevalence and characteristics of nodular anterior mediastinal lesions that were found incidentally on screening chest CT in asymptomatic subjects (1). In the latter study, nodular lesions were incidentally detected in the anterior mediastinum in 413 [0.73\% (95\% confidence interval: $0.66-0.80 \%)]$ of the 56,358 consecutive participants. In addition, the prevalence of such lesions increased with age $(\mathrm{P}<0.001)$, e.g., the prevalence of such nodular lesions at age $25-34$ years was $0.4 \%$, while at $65-74$ years and $\geq 75$ years it was $1.26 \%$ and $1.44 \%$, respectively. The CT features of these lesions were also evaluated in the latter study. Most $(85.2 \%)$ of the lesions were small (long-axis diameter: $<2.0 \mathrm{~cm}$ ). The median long-axis and short-axis diameters of the lesions were 1.2 and $0.8 \mathrm{~cm}$, respectively. Almost all $(97.8 \%)$ of the lesions exhibited well defined margins, and $61.3 \%$ of the lesions were round. The median CT attenuation value of the lesions was 43 Hounsfield units
(HU), and $80.2 \%$ of the lesions displayed CT attenuation values of $>20 \mathrm{HU}$, which indicated that they were not cystic lesions. Among the 51 cases in which final diagnoses were confirmed, about $23 \%$ of the lesions were malignant (almost all of which were TET, and about $90 \%$ of which were classified as Masaoka stage I or II), while the remaining 77\% of lesions were benign (about $80 \%$ of which were thymic cysts). This study also investigated the changes in the sizes of unconfirmed lesions seen on follow-up CT. During the follow-up period (median duration: 50.9 months), the sizes of $82.2 \%$ of the lesions remained stable. In summary, anterior mediastinal lesions that were found incidentally on CT had imaging features that were indistinguishable from those of TET. However, many of the lesions seemed to be benign, and many of the TET were early-stage lesions.

The aforementioned study examined a larger population than previous studies $(2,3)$. In addition, it also included some cases $(12.3 \%$ of incidental anterior thymic lesions) that were diagnosed pathologically. Even in the pathologically unconfirmed cases, a lot of follow-up data were reported. Such important information could help to prevent patients with incidentally detected benign thymic lesions from having to undergo unnecessary thymectomy procedures and might also provide physicians with clues about the appropriate management of such lesions.

The study by Yoon et al. (1) did not focus on the differences between the CT features of malignant and benign lesions. The incidentally detected anterior mediastinal nodular lesions tended to be relatively small, 
and the benign lesions resembled thymic malignancies. However, imaging-based differential diagnosis can facilitate the management of such anterior mediastinal lesions. Although the CT findings of anterior mediastinal lesions overlap to some extent, there are some characteristic findings that are useful for differentiating among such lesions. Various CT findings that are useful for differentiating between benign thymic lesions and TET have been reported (4-6). The non-therapeutic thymectomy rate (i.e., thymectomy for lymphoma or benign disease) at a single institution was reported to be about $44 \%$ (lymphoma: $54.3 \%$, thymic bed cysts: $24.3 \%$ ) (4). This high rate of unnecessary thymectomy was caused by the difficulty of differentiating these diseases from TET on CT. The role of imaging in solving such problems should be investigated further, although there is overlap in the imaging features of these diseases. Magnetic resonance imaging (MRI) is a potentially useful technique for evaluating incidental anterior mediastinal lesions, especially those that are suspected to be cystic lesions, like thymic cysts, on CT, e.g., well circumscribed, round, oval or saccular lesions or nodular lesions that exhibit homogenous attenuation and are located near the thymic bed $(4,6,7)$. Cystic lesions typically exhibit hypointensity or hyperintensity on T1weighted imaging (T1WI) and hyperintensity on T2WI, and this information could help physicians to differentiate between indeterminate thymic cysts and solid nodules, especially due to their relatively high attenuation values. Furthermore, MRI is superior to CT at depicting contrastenhanced or soft tissue components. Thymic hyperplasia typically presents as a triangular or quadrilateral lesion on CT and tends to arise along the midline of the thymic bed. Yoon et al. (1) excluded such lesions from their study population. In cases in which it is difficult to differentiate thymic hyperplasia from true nodular or massive lesions, chemical shift MRI can be used to detect the presence of intralesional fat tissue (8).

Evaluating the doubling time of incidentally detected anterior mediastinal lesions is also important for managing such lesions, and an article about the doubling time of TET and the association between the doubling times of such lesions on CT and their histological subtype has been reported (9). The latter article focused on TET, which represent the main clinical concern in the anterior mediastinum. According to two-dimensional evaluations, the median doubling time of TET was 400 days, but varied markedly (range, 48-1,964 days). On the other hand, the median doubling times of low-risk thymoma
(WHO classification type $\mathrm{A}, \mathrm{AB}$, and $\mathrm{B} 1$ ), high-risk thymoma (type B2, and B3), and thymic carcinoma were 436, 381, and 189 days, respectively, and the doubling times of low-risk thymoma and thymic carcinoma were significantly different, as were those of high-risk thymoma and thymic carcinoma. Type B3 thymoma and thymic carcinoma were found to be associated with rapid growth (doubling time: $<180$ days). So, TET that exhibit doubling times of $<180$ days should be considered aggressive and require active management. Changes in the shapes of anterior mediastinal nodules on follow-up CT can also provide useful information because thymic cysts sometimes display such findings. Lesion size is a predictor of malignancy and might provide useful information for follow-up planning. For example, the proportion of malignant lesions tends to be significantly higher among lesions of $>2 \mathrm{~cm}$ in diameter than among those of $\leq 2 \mathrm{~cm}$ in diameter (1).

Age is also an important factor for predicting malignancy and differential diagnosis. In patients aged $<43$ years, indeterminate anterior mediastinal lesions tend to be benign (5), although it has been reported that in the anterior mediastinum malignant germ cell tumors and malignant lymphoma are more common than TET in young patients (10), and attention should be paid to the risk of such malignant lesions in young patients. Finally, elevated lactate dehydrogenase, alpha-fetoprotein, and beta-human chorionic gonadotropin levels can sometimes be helpful for differentiating among anterior mediastinal lesions.

In conclusion, various chest CT findings of incidental anterior mediastinal nodular lesions can help physicians to manage such lesions in an appropriate manner. Furthermore, it is hoped that a clinically useful algorithm for guiding the management of such lesions will be established, e.g., such an algorithm could help to optimize the follow-up period and follow-up intervals based on diagnostic imaging criteria; the lesion's doubling time; and other clinical information, such as the patient's age and laboratory data.

\section{Acknowledgements}

None.

\section{Footnote}

Conflicts of Interest: The author has no conflicts of interest to declare. 


\section{References}

1. Yoon SH, Choi SH, Kang CH, et al. Incidental Anterior Mediastinal Nodular Lesions on Chest CT in Asymptomatic Subjects. J Thorac Oncol 2018;13:359-66.

2. Henschke CI, Lee IJ, Wu N, et al. CT screening for lung cancer: prevalence and incidence of mediastinal masses. Radiology 2006;239:586-90.

3. Araki T, Nishino M, Gao W, et al. Anterior Mediastinal Masses in the Framingham Heart Study: Prevalence and CT Image Characteristics. Eur J Radiol Open 2015;2:26-31.

4. McErlean A, Huang J, Zabor EC, et al. Distinguishing benign thymic lesions from early-stage thymic malignancies on computed tomography. J Thorac Oncol 2013;8:967-73.

5. Ackman JB, Verzosa S, Kovach AE, et al. High rate of unnecessary thymectomy and its cause. Can computed

doi: 10.21037/shc.2018.11.09

Cite this article as: Ozawa Y. The management of anterior mediastinal nodules that are incidentally found on CT. Shanghai Chest 2018;2:92. tomography distinguish thymoma, lymphoma, thymic hyperplasia, and thymic cysts? Eur J Radiol 2015;84:524-33.

6. Carter BW, Okumura M, Detterbeck FC, et al. Approaching the patient with an anterior mediastinal mass: a guide for radiologists. J Thorac Oncol 2014;9:S110-8.

7. Inaoka T, Takahashi K, Mineta M, et al. Thymic hyperplasia and thymus gland tumors: differentiation with chemical shift MR imaging. Radiology 2007;243:869-76.

8. Choe J, Lee SM, Lim S, et al. Doubling time of thymic epithelial tumours on CT: correlation with histological subtype. Eur Radiol 2017;27:4030-6.

9. Araki T, Sholl LM, Gerbaudo VH, et al. Intrathymic cyst: clinical and radiological features in surgically resected cases. Clin Radiol 2014;69:732-8.

10. Carter BW, Marom EM, Detterbeck FC. Approaching the patient with an anterior mediastinal mass: a guide for clinicians. J Thorac Oncol 2014;9:S102-9. 